\title{
A Legendre wavelet spectral collocation method for analysis of thermal radiation and slip in the oblique stagnation-point flow of Walters-B liquid towards a stretching surface
}

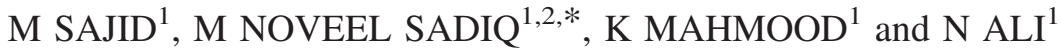 \\ ${ }^{1}$ Department of Mathematics and statistics, International Islamic University, Islamabad 44000, Pakistan \\ ${ }^{2}$ School of Natural Sciences, National University of Sciences and Technology, H-12, Islamabad, Pakistan \\ e-mail: noveelsheikh@gmail.com
}

MS received 31 July 2018; revised 26 December 2018; accepted 5 February 2019; published online 6 May 2019

\begin{abstract}
A spectral collocation method based on Legendre wavelet is applied to analyse the slip and radiation effects in the flow of an elastico-viscous Walters-B fluid. The fluid is assumed to impinge obliquely on a stretching surface with linear stretching velocity. The governing system of equations is converted into dimensionless ordinary differential equations by invoking suitable similarity variables. The proposed iterative spectral technique for solving governing system of initial value problems is applied for obtaining the solutions. First, we extend Legendre wavelet and Legendre-Guass collocation points are computed for a large interval. The differential equations are converted into a system of algebraic equations. The approximate solution is obtained by solving these algebraic equations. The proposed algorithm is implemented to obtain numerical results for different values of the pertinent parameters. The proposed algorithm controlled the overshooting and undershooting in velocity profiles. The presence of stretching and slip enhances the velocity and reduces the temperature of the fluid within the boundary layer.
\end{abstract}

Keywords. Stretching sheet; viscoelastic fluid; oblique stagnation point flow; thermal radiation; Legendre wavelet spectral collocation method; shooting method.

\section{Introduction}

Recent years saw a remarkable growth in the literature regarding non-Newtonian fluids. These fluids obey nonlinear constitutive relationship between the shear stress and deformation rate and have practical applications in chemical, metallurgical, civil and mining engineering and industries. There exist various constitutive relationships of non-Newtonian fluids in the literature. Among these the Walters-B [1] fluid is an important viscoelastic fluid. The nonlinearity and higher-order derivatives restrict the researchers to solve flow equations of Walters-B fluid by conventional methods. Moreover, there exists a strong singularity at the initial boundary. This complexity challenged mathematicians to develop methods that overcome this difficulty. Beard and Walters [1] used the first-order perturbation method to find the solution. The perturbation solution predicted the velocity overshoot within the boundary layer. Frater [2] was of the opinion that perturbation solution is responsible for overshoot in the velocity profile. Ariel [3] proposed a hybrid numerical technique to explain the stagnation-point flows of Walters-B liquid and

*For correspondence reported the overshoot in the velocity. Dorrepaal et al [4] discussed the flow of Walters-B fluid near point of reattachment.

The flow over a stretching surface has frequent engineering applications such as polymer extrusion, artificial fibres and rolling and manufacturing of plastic films. In the process of melt-spinning, the extrudate from the die is usually drawn and at the same time stretched into a sheet, which is converted into a solid by gradual cooling process. The heat transfer at stretching surface influences the quality of the final product. Crane [5] investigated the attached flow with a stretching surface utilizing the boundary layer equations of a viscous fluid. McLeod and Rajagopal [6] studied the unique solution of a viscous fluid flow past a stretchable boundary. Mahapatra et al [7] explored the flow of Walters-B fluid near the stagnated region over a flat stretching surface. Lok et al [8] studied the non-orthogonal stagnated flow of a viscous fluid near a stretching sheet. Hussain et al [9] deliberated the stagnated flow of WaltersB liquid impinging obliquely over a stretching surface. Hayat et al [10] explored the three-dimensional flow of an elastico-viscous fluid over a stretching surface. Wang [11] analytically solved the problem of slip flow of a viscous fluid near the stagnation region. Labropulu et al [12] 
evaluated the slip effects in the stagnation-region of Walters-B liquid. Sajid et al [13] investigated the axisymmetric flow near a stagnation-point over a lubricated disk with power-law fluid as lubricant by introducing generalized slip boundary condition. Mehmood et al [14-17] considered the flow of an elastico-viscous fluid, micropolar, couple stress and Jeffrey fluids, respectively, towards an oblique stagnation point caused by a lubricated flat surface. Kushwaha and Sahu [18] discussed the heat transfer in the presence of the slip in the region between two heated parallel plates having the effects of viscous dissipation. Zhu et al [19] evaluated the effects of the velocity slip of the second order on the nanofluid flow in the presence of MHD.

In the last few years, attention has been paid to heat transfer in non-Newtonian fluids in many engineering and industrial activities, e.g. food processing, pharmaceutical process, hybrid power engines, microelectronics, fuel cells, hyperthermia, coolants in nuclear power plants, etc. Dandapat and Gupta [20] analysed the heat transfer in secondgrade fluid over a stretchable surface. Sajid et al [21] discussed the micropolar fluid flow over a disk with combined effects of uniform rotation and linear radial stretching. Labropulu et al [22] calculated the heat transfer in oblique flow of a viscoelastic fluid towards a stretchable surface. The analysis was made by considering the non-Newtonian fluid in the oblique stagnation zone. Reza et al [23] discussed the heat transfer in stagnation flow of a elasticoviscous fluid impinging on a quiescent fluid. Hayat et al [24] studied the radiative and convective heat transfer phenomenon in Walters-B liquid flow. Hakeem et al [25] analysed the effects of thermal radiation and elastic deformation in Walters-B fluid. Ali et al [26] examined heat transfer due to thermal radiation and heat source/sink in a unsteady flow of a non-Newtonian fluid past an oscillatory stretching sheet. Seedak and Abdelmeguid [27] investigated the effects of thermal radiation with thermal diffusivity over a stretching sheet having variable heat flux. Babu and Sandeep [28], Raju and Sandeep [29] and Kumar et al [30] focused on the effects of non-linear thermal radiations by incorporating the Newtonian and non-Newtonian fluids in the presence and absence of the slip effects. Attia et al [31] and Prakashi et al [32] investigated the heat transfer in fluid flow between two porous plates and the porous channel.

The spectral methods are widely used to deal with the nonlinearity of a system of equations. Yuan et al [34] introduced the multidomain pseudo-spectral approximation based on the Legendre-Galerkin method. They solved nonlinear diffusion equations to check the stability and the rate of convergence of the method.

Keeping in view this literature, the current investigation is performed to predict the effects of the slip and thermal radiation on the oblique two-dimensional flow of Walters-B fluid near a stagnation point with uniform heat source/sink. Governing equations are solved mathematically using a numerical method named as Legendre wavelet spectral collocation method (LWSCM) proposed by Sajid et al [35]. No overshoot in the velocity reported in the work of Arial [3] and Hussain et al [9] is seen while applying the LWSCM numerical technique.

\section{Problem formulation}

A steady, two-dimensional and incompressible oblique stagnation-point flow of Walters-B fluid over a stretchable sheet at $y=0$ is considered (figure 1). The flow and heat transfer are studied by following [1, 9, 25].

$$
\begin{gathered}
\frac{\partial \bar{u}}{\partial \bar{x}}+\frac{\partial \bar{v}}{\partial \bar{y}}=0 \\
\bar{u} \frac{\partial \bar{u}}{\partial \bar{x}}+\bar{v} \frac{\partial \bar{u}}{\partial \bar{y}}=-\frac{1}{\rho} \frac{\partial \bar{p}}{\partial \bar{x}}+v \bar{\nabla}^{2} \bar{u} \\
-\frac{k_{0}}{\rho}\left[\frac { \partial } { \partial \overline { x } } \left[2 \bar{u} \frac{\partial^{2} \bar{u}}{\partial \bar{x}^{2}}+4\left(\frac{\partial \bar{u}}{\partial \bar{x}}\right)^{2}+2 \bar{v} \frac{\partial^{2} \bar{u}}{\partial \bar{x} \partial \bar{y}}\right.\right. \\
\left.+2 \frac{\partial \bar{v}}{\partial \bar{x}}\left(\frac{\partial \bar{v}}{\partial \bar{x}}+\frac{\partial \bar{u}}{\partial \bar{y}}\right)\right]+\frac{\partial}{\partial \bar{y}}\left[\left(\bar{u} \frac{\partial}{\partial \bar{x}}+\bar{v} \frac{\partial}{\partial \bar{y}}\right)\left(\frac{\partial \bar{v}}{\partial \bar{x}}+\frac{\partial \bar{u}}{\partial \bar{y}}\right)\right. \\
\left.\left.+2 \frac{\partial \bar{v}}{\partial \bar{x}} \frac{\partial \bar{v}}{\partial \bar{y}}+2 \frac{\partial \bar{u}}{\partial \bar{x}} \frac{\partial \bar{u}}{\partial \bar{y}}\right]\right], \\
\bar{u} \frac{\partial \bar{v}}{\partial \bar{x}}+\bar{v} \frac{\partial \bar{v}}{\partial \bar{y}}=-\frac{1}{\rho} \frac{\partial \bar{p}}{\partial \bar{y}}+v \bar{\nabla}^{2} \bar{v} \\
\quad-\frac{k_{0}}{\rho}\left[\frac { \partial } { \partial \overline { x } } \left[\left(\bar{u} \frac{\partial}{\partial \bar{x}}+\bar{v} \frac{\partial}{\partial \bar{y}}\right)\left(\frac{\partial \bar{v}}{\partial \bar{x}}+\frac{\partial \bar{u}}{\partial \bar{y}}\right)+2 \frac{\partial \bar{u}}{\partial \bar{x}} \frac{\partial \bar{u}}{\partial \bar{y}}\right.\right. \\
\left.+2 \frac{\partial \bar{v}}{\partial \bar{x}} \frac{\partial \bar{v}}{\partial \bar{y}}\right]+\frac{\partial}{\partial \bar{y}}\left[2 \bar{u} \frac{\partial^{2} \bar{v}}{\partial \bar{x} \partial \bar{y}}+2 \bar{v} \frac{\partial^{2} \bar{v}}{\partial \bar{y}^{2}}+4\left(\frac{\partial \bar{v}}{\partial \bar{y}}\right)^{2}\right. \\
\left.\left.+2 \frac{\partial \bar{u}}{\partial \bar{y}}\left(\frac{\partial \bar{v}}{\partial \bar{x}}+\frac{\partial \bar{u}}{\partial \bar{y}}\right)\right]\right] \\
+c_{p}^{2} T \\
+\frac{1}{\rho c_{p}} \frac{\partial q_{r}}{\partial \bar{y}}+\frac{Q}{\rho c_{p}}\left(T-T_{\infty}\right)
\end{gathered}
$$

where $u(x, y)$ and $v(x, y)$, respectively, are components of velocity in $x$ and $y$ directions; $v, \rho, c_{p}, k, q_{r}$ and $Q$,

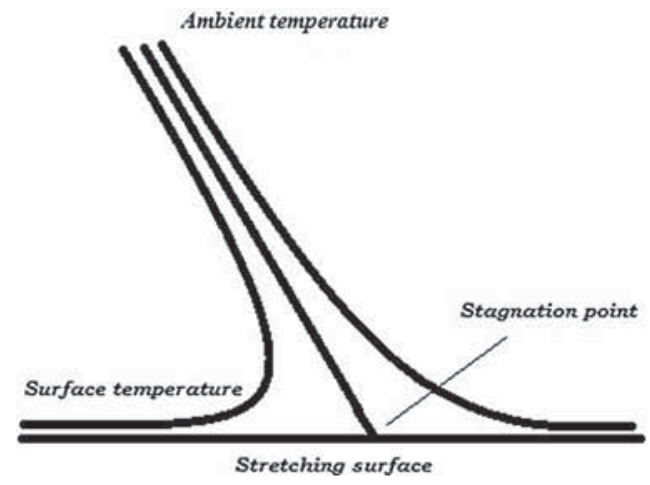

Figure 1. Flow geometry of physical problem. 
respectively, are kinematic viscosity, density, specific heat, thermal conductivity, radiative heat flux and the heat source/sink. The Rossland approximation $q_{r}=\frac{-4 \sigma^{*}}{3 k^{*}} \frac{\partial T^{4}}{\partial \bar{y}}$ where $\sigma^{*}$ and $k^{*}$, respectively, are the Stefan-Boltzmann constant and the mean absorption coefficient is used. Furthermore, $T, T_{w}$ and $T_{\infty}$, respectively, are the temperature of fluid inside boundary layer, at the wall and far away from the wall. Defining stream function from (1) such that

$$
\bar{u}=\frac{\partial \bar{\psi}}{\partial \bar{y}}, \quad \bar{v}=-\frac{\partial \bar{\psi}}{\partial \bar{x}} .
$$

Substituting (5) into (2)-(4) and eliminating the pressure we have

$$
\begin{gathered}
\frac{\partial\left(\bar{\psi}, \nabla^{2} \bar{\psi}\right)}{\partial(\bar{x}, \bar{y})}+\frac{k_{0}}{\rho} \frac{\partial\left(\bar{\psi}, \nabla^{4} \bar{\psi}\right)}{\partial(\bar{x}, \bar{y})}+v \nabla^{4} \bar{\psi}=0 \\
\frac{\partial \bar{\psi}}{\partial \bar{y}} \frac{\partial T}{\partial \bar{x}}+\frac{\partial \bar{\psi}}{\partial \bar{x}} \frac{\partial T}{\partial \bar{y}}=\frac{k}{\rho c_{p}} \frac{\partial^{2} T}{\partial \bar{y}^{2}}-\frac{1}{\rho c_{p}} \frac{\partial q_{r}}{\partial \bar{y}}+\frac{Q}{\rho c_{p}}\left(T-T_{\infty}\right) .
\end{gathered}
$$

The appropriate boundary conditions are [9]

$$
\begin{gathered}
\bar{\psi}=0, \quad \frac{\partial \bar{\psi}}{\partial \bar{y}}=c \bar{x}+\left.\frac{b}{\mu} \tau^{12}\right|_{\bar{y}=0}, \quad T=T_{w}+\alpha^{*} \frac{\partial T}{\partial \bar{y}} \\
\text { at } \quad \bar{y}=0, \\
\bar{\psi}=a \bar{x} \bar{y}+\frac{1}{2} b \bar{y}^{2}, \quad T \rightarrow T_{\infty} \quad \text { as } \quad \bar{y} \rightarrow \infty,
\end{gathered}
$$

where $a, b$ and $c$ are constants and $\alpha^{*}$ is thermal slip parameter. The heat transfer rate and skin friction coefficient at wall can be expressed as [26]

$$
C_{f}=\frac{\tau_{w}}{\rho(c \bar{x})^{2}}, \quad N u_{x}=\frac{\bar{x} q_{w}}{k\left(T_{w}-T_{\infty}\right)},
$$

where

$$
\begin{gathered}
\tau_{w}=\left\{\mu\left(\frac{\partial^{2} \bar{\psi}}{\partial \bar{y}^{2}}-\frac{\partial^{2} \bar{\psi}}{\partial \bar{x}^{2}}\right)-k_{o}\left[\frac{\partial \bar{\psi}}{\partial \bar{y}}\left(\frac{\partial^{3} \bar{\psi}}{\partial \bar{x} \partial \bar{y}^{2}}-\frac{\partial^{3} \bar{\psi}}{\partial \bar{x}^{3}}\right)\right.\right. \\
\left.\left.-\frac{\partial \bar{\psi}}{\partial \bar{x}}\left(\frac{\partial^{3} \bar{\psi}}{\partial \bar{y}^{3}}-\frac{\partial^{3} \bar{\psi}}{\partial \bar{x}^{2} \partial \bar{y}}\right)+2 \bar{\psi}_{\bar{x} \bar{y}} \bar{\psi}_{\bar{x} \bar{x}}+2 \bar{\psi}_{\bar{y} \bar{y}} \bar{\psi}_{\bar{x} \bar{y}}\right]\right\}_{\bar{y}=0} . \\
q_{w}=-k\left(1+\frac{16 \sigma^{*} T_{\infty}^{3}}{3 k k^{*}}\right)\left(\frac{\partial T}{\partial \bar{y}}\right)_{\bar{y}=0} .
\end{gathered}
$$

Defining dimensionless variables

$$
x=\bar{x} \sqrt{\frac{c}{v}}, \quad y=\bar{y} \sqrt{\frac{c}{v}}, \quad \psi=\frac{\bar{\psi}}{v},
$$

Eqs. (6)-(9) in dimensionless variables become

$$
\frac{\partial\left(\psi, \nabla^{2} \psi\right)}{\partial(x, y)}+W e \frac{\partial\left(\psi, \nabla^{4} \psi\right)}{\partial(x, y)}+v \nabla^{4} \psi=0
$$

$$
\begin{gathered}
\frac{\partial \psi}{\partial y} \frac{\partial T}{\partial x}+\frac{\partial \psi}{\partial x} \frac{\partial T}{\partial y}=\frac{k}{\rho c_{p}} \frac{\partial^{2} T}{\partial y^{2}}-\frac{1}{\rho c_{p}} \frac{\partial q_{r}}{\partial y}+\frac{Q}{\rho c_{p}}\left(T-T_{\infty}\right), \\
\psi=0, \quad \frac{\partial \psi}{\partial y}=x+\left.\beta \tau^{12}\right|_{y=0}, \quad T=T_{w}+\alpha \frac{\partial T}{\partial y} \\
\text { at } \quad y=0, \\
\psi=\frac{a}{c} x y+\frac{1}{2} \gamma y^{2}, \quad T \rightarrow T_{\infty} \quad \text { as } \quad y \rightarrow \infty
\end{gathered}
$$

where $W e=\frac{k_{0} c}{\rho v}, \gamma=\frac{b}{c}, \beta=b \sqrt{\frac{c}{v}}$ and $\alpha=\alpha^{*} \sqrt{\frac{c}{v}}$, respectively, are viscoelastic parameter Weissenberg number, shear in freestream, velocity and thermal slip parameters. Introduce the similarity transformations

$$
\psi(x, y)=x F(y)+G(y), \quad \theta(y)=\frac{T-T_{\infty}}{T_{w}-T_{\infty}},
$$

where $F(y)$ and $G(y)$, respectively, denote the component of the flow in normal and tangential directions. Applying Eq. (18) into Eqs. (14)-(17) and after integration, the following system of ordinary differential equations is obtained:

$$
\begin{aligned}
& F^{\prime \prime \prime}(y)-\left[F^{\prime}(y)\right]^{2}+F(y) F^{\prime \prime}(y)+\frac{a^{2}}{c^{2}}+W e\left[F(y) F^{i v}(y)\right. \\
& \left.+F^{\prime \prime \prime}(y)+\left[F^{\prime \prime}(y)\right]^{2}-2 F^{\prime}(y)\right]=0,
\end{aligned}
$$

$F(0)=0, \quad F^{\prime}(0)=\frac{1+\beta F^{\prime \prime}(0)}{3 \beta W e F^{\prime \prime}(0)+1}, \quad F^{\prime}(\infty)=\frac{a}{c}$,

$$
\begin{aligned}
& G(0)=0, \quad G^{\prime}(0) \\
& \quad=\frac{\beta G^{\prime \prime}(0)}{1+\beta W e F^{\prime \prime}(0)}\left[1-2 W e\left(\frac{1+\beta F^{\prime \prime}(0)}{3 \beta W e F^{\prime \prime}(0)+1}\right)\right], \\
& G^{\prime \prime}(\infty)=\gamma,
\end{aligned}
$$

$$
\theta(0)=1+\alpha \theta^{\prime}(0), \quad \theta(\infty)=0,
$$

where $A=A(W e, a / c)$ is the boundary layer displacement and $P r_{\text {eff }}=\frac{P r}{N r+1}$ denotes the effective Prandtl number. It was the opinion of the Magyari and Pantokratoras [33] that energy equation (24) should not be solved by an approach using two parameters, i.e., Prandtl number and radiation 
parameter. They disclosed that in fact the study of heat transfer characteristics has similar consequences either with or without thermal radiation. It was emphasized that the flow problems involving thermal radiations admit the same solution for infinite parametric values of $(\mathrm{Nr}, \mathrm{Pr})$, which corresponds to the same effective Prandtl number. Equation (20) can be further simplified as

$$
\begin{gathered}
G^{\prime}(y)=\gamma H(y) \\
H^{\prime \prime}(y)+H^{\prime}(y) F(y)-H(y) F^{\prime}(y) \\
+W e\left[H^{\prime \prime \prime}(y) F(y)-H^{\prime \prime}(y) F^{\prime}(y)\right. \\
\left.+H^{\prime}(y) F^{\prime \prime}(y)-H(y) F^{\prime \prime \prime}(y)\right]=A \\
H(0)=\frac{\beta H^{\prime}(0)}{1+\beta W e F^{\prime \prime}(0)}\left[1-2 W e\left(\frac{1+\beta F^{\prime \prime}(0)}{3 \beta W e F^{\prime \prime}(0)+1}\right)\right] \\
H^{\prime}(\infty)=1
\end{gathered}
$$

Employing Eq. (18) in Eq. (10), we have

$$
\begin{gathered}
\operatorname{Re}_{x} C_{f}=x F^{\prime \prime}(0)(1-3 W e)+\gamma H^{\prime}(0)(1-2 W e), \\
R e_{x}^{-1 / 2} N u_{x}^{*}=-\theta^{\prime}(0),
\end{gathered}
$$

where $N u_{x}^{*}=\frac{N u_{x}}{(1+N r)}$ is local effective Nusselt number.

\section{LWSCM}

Consider the interval $[0, T)$. The Legendre wavelets are defined as

$$
\begin{aligned}
& \psi_{m, n}(t)= \\
& \begin{cases}\left(m+\frac{1}{2}\right)^{1 / 2} 2^{k / 2} L_{m}\left(2^{k} t-2 n+1\right), & \frac{n-1}{2^{k-1}} \leq t<\frac{n}{2^{k-1}} \\
0, & \text { otherwise. }\end{cases}
\end{aligned}
$$

$L_{m}(t)$ is $m$ th-order Legendre polynomial having $w(t)=1$ orthogonal weight function. One can obtain the Legendre polynomials using the relations

$$
\begin{aligned}
L_{0}(t) & =1, \\
L_{1}(t) & =t \\
L_{m+1}(t) & =\frac{2 m+1}{m+1} t L_{m}(t)-\frac{m}{m+1} L_{m-1}(t), \quad m=1,2,3 \ldots
\end{aligned}
$$

$x_{0}<x_{1}<x_{2} \cdots<x_{m-1}$, known as Legendre-Gauss collocation points, are roots of the $L_{m}(x)$ in $(-1,1)$ and $\left\{w_{j}\right\}_{j=0}^{m-1}$ are corresponding weights:

$$
w_{j}=\frac{2}{\left(1-x_{j}^{2}\right)\left(L_{M}^{\prime}\left(x_{j}\right)\right)^{2}}, \quad j=0,1, \ldots, M-1 .
$$

Any function $f \in L^{2}\left[0, y_{\infty}\right)$ in terms of Legendre wavelets takes the form

$$
f(x)=\sum_{n=1}^{\infty} \sum_{m=0}^{\infty} y_{n, m} \psi_{n, m}(x)
$$

where $y_{n, m}$ can be approximated to

$$
y_{n, m} \simeq \sum_{j=0}^{M-1} \bar{w}_{j} f\left(x_{n j}\right) \psi_{n, m}\left(x_{n j}\right)
$$

where

$$
\begin{gathered}
\bar{w}_{j}=\frac{w_{j}}{2^{k}}, \quad x_{n j}=\frac{x_{j}}{2^{k}}+\frac{2 n-1}{2^{k}}, \\
n=1,2, \ldots, 2^{k-1} T, \quad j=0,1, \ldots M-1 .
\end{gathered}
$$

Hence, Eq. (33) becomes

$$
\begin{aligned}
f(x) \simeq & \sum_{n=1}^{\infty} \sum_{m=0}^{\infty} \sum_{j=0}^{M-1} \bar{w}_{j} f\left(x_{n j}\right) \psi_{n, m}\left(x_{n j}\right) \psi_{n, m}(x) . \\
& \sum_{n=1}^{\infty} \sum_{m=0}^{\infty} \psi_{n, m}(x) \psi_{n, m}(t)=\delta(x-t)
\end{aligned}
$$

is an identity. Truncating this to $M-1$ and $2^{k-1} T$ and introducing

$$
I_{n j}(x)=\sum_{m=0}^{M-1} \bar{w}_{j} \psi_{n, m}\left(x_{n j}\right) \psi_{n, m}(x),
$$

we have

$$
f(x) \simeq \sum_{n=1}^{2^{k-1}} \sum_{j=0}^{M-1} I_{n j}(x) f\left(x_{n j}\right)
$$

\subsection{Solution by LWSCM}

Consider the boundary value problems (19)-(24). We convert boundary value problems into initial value problems by assuming

$$
F^{\prime \prime}(0)=s, \quad G^{\prime \prime}(0)=r, \quad \theta^{\prime}(0)=t .
$$

To obtain an approximate solution of these problems, we divide the domain $0<y<T$ into subintervals for $n=$ $1, \ldots, 2^{k-1} T$ by $\left[\frac{n-1}{2^{k-1}}, \frac{n}{2^{k-1}}\right)$. The functions $F(y)$ and $Z(y)$ can be approximated using Legendre wavelet interpolation on the $n$th subinterval as

$$
F(y) \simeq \sum_{n=1}^{2^{k-1} T} F_{n}(y)=\sum_{n=1}^{2^{k-1} T} \sum_{j=0}^{M-1} I_{n j}(y) F\left(y_{n j}\right),
$$




$$
\begin{aligned}
G(y) & \simeq \sum_{n=1}^{2^{k-1} T} G_{n}(y)=\sum_{n=1}^{2^{k-1}} \sum_{j=0}^{M-1} I_{n j}(y) G\left(y_{n j}\right), \\
\theta(y) & \simeq \sum_{n=1}^{2^{k-1} T} \theta_{n}(y)=\sum_{n=1}^{2^{k-1}} \sum_{j=0}^{M-1} I_{n j}(y) \theta\left(y_{n j}\right),
\end{aligned}
$$

and the derivatives can be written as

$$
\begin{aligned}
& F^{m}(y) \simeq \sum_{n=1}^{2^{k-1} T} F_{n}^{m}(y)=\sum_{n=1}^{2^{k-1} T} \sum_{j=0}^{M-1} I_{n j}^{m}(y) F\left(y_{n j}\right), \\
& Z^{m}(y) \simeq \sum_{n=1}^{2^{k-1} T} Z_{n}^{m}(y)=\sum_{n=1}^{2^{k-1} T} \sum_{j=0}^{M-1} I_{n j}^{m}(y) Z\left(y_{n j}\right), \\
& \theta^{m}(y) \simeq \sum_{n=1}^{2^{k-1} T} \theta_{n}^{m}(y)=\sum_{n=1}^{2^{k-1} T} \sum_{j=0}^{M-1} I_{n j}^{m}(y) \theta\left(y_{n j}\right) .
\end{aligned}
$$

Applying the points $\left\{y_{n j} \mid n=1, \ldots, 2^{k-1} T, j=\right.$ $3, \ldots, M-1\}$ into Eqs. (19)-(24), we obtain

$$
F_{n}^{\prime \prime \prime}+F_{n}^{\prime \prime} F_{n}-F_{n}^{\prime 2}+\frac{a^{2}}{c^{2}}+W e\left[F_{n} F_{n}^{i v}-2 F_{n}^{\prime \prime \prime} F_{n}^{\prime}+F_{n}^{\prime \prime 2}\right]=0
$$

$$
\begin{gathered}
G_{n}^{\prime \prime \prime}+G_{n}^{\prime \prime} F_{n}-G_{n}^{\prime} F_{n}^{\prime} \\
+W e\left[G_{n}^{i v} F_{n}-G_{n}^{\prime \prime \prime} F_{n}^{\prime}+G_{n}^{\prime \prime} F_{n}^{\prime \prime}-G_{n}^{\prime} F_{n}^{\prime \prime \prime}\right]=A \gamma \\
\theta_{n}^{\prime \prime}+\operatorname{Pr}_{\text {eff }} \theta_{n}^{\prime} F_{n}+\lambda P r_{\text {eff }} \theta_{n}=0 \\
F_{n}(0)=0, \quad F_{n}^{\prime}(0)=\frac{1+\beta F_{n}^{\prime \prime}(0)}{3 \beta W e F_{n}^{\prime \prime}(0)+1}, \quad F_{n}^{\prime \prime}(0)=s,
\end{gathered}
$$

$$
\begin{gathered}
G_{n}(0)=0, \quad G_{n}^{\prime}(0)=\frac{\beta G_{n}^{\prime \prime}(0)}{1+\beta W e F_{n}^{\prime \prime}(0)} \\
{\left[1-2 W e\left(\frac{1+\beta F_{n}^{\prime \prime}(0)}{3 \beta W e F_{n}^{\prime \prime}(0)+1}\right)\right],} \\
G_{n}^{\prime \prime}(0)=r,
\end{gathered}
$$

Table 1. Comparison of the results for $\beta=0$.

$$
\theta_{n}(0)=1+\alpha \theta_{n}^{\prime}(0), \quad \theta_{n}^{\prime}(0)=t .
$$

An approximate value is chosen for $s, r$ and $t$ and the system of Eqs. (48)-(53) is solved for the first subinterval, i.e. $\left[0,1 / 2^{k-1}\right)$. The results of first subinterval are used as initial guess for second subinterval and this process is repeated for all subintervals. A root finding algorithm that satisfies $F^{\prime}(\infty)=a / c, G^{\prime \prime}(\infty)=\gamma$ and $\theta(\infty)=0$ is applied to modify the values of $s, r$ and $t$, respectively.

\section{Results and discussion}

To find the solution of the problem, a numerical technique known as LWSCM is applied. A comparison of numerical data is made using available literature of Hussain et al [9] for no-slip boundary condition as shown in table 1. A deep analysis of this table demonstrates that the method has excellent agreement for the viscous case, and as the viscoelastic parameter increases the difference with the numerical data occurs. This is because of the undershoot and overshoot in the velocity profiles within the boundary layer observed in the work of Hussain et al [9]. However, the method implemented in this article has the ability to control the overshooting and undershooting phenomenon while solving these equations, which gives more accurate and stable solutions. Secondly, there is no limitation on the viscoelastic parameter by employing LWSCM; it is not possible to obtain the solutions beyond the critical value of

\begin{tabular}{|c|c|c|c|c|c|c|c|c|}
\hline \multirow{2}{*}{$\begin{array}{l}\text { We } \\
-\end{array}$} & \multicolumn{2}{|c|}{$a / c=0.1$} & \multicolumn{2}{|c|}{$a / c=0.5$} & \multicolumn{2}{|c|}{$a / c=1.1$} & \multicolumn{2}{|c|}{$a / c=1.2$} \\
\hline & [9] & LWSCM & [9] & LWSCM & [9] & LWSCM & [9] & LWSCM \\
\hline 0.0 & -0.9693 & -0.969478 & -0.6672 & -0.667284 & 0.1642 & 0.164293 & 0.3377 & 0.337744 \\
\hline 0.05 & -0.9971 & -0.997033 & -0.6967 & -0.696570 & 0.17646 & 0.176353 & 0.3646 & 0.364366 \\
\hline 0.1 & -1.0271 & -1.026342 & -0.7299 & -0.728904 & 0.19177 & 0.191053 & 0.3992 & 0.397446 \\
\hline 0.2 & -1.0955 & -1.091446 & -0.8101 & -0.804906 & 0.2392 & 0.233221 & 0.5139 & 0.496825 \\
\hline 0.3 & -1.1777 & -1.168468 & -0.9141 & -0.901446 & 0.35198 & 0.312576 & 0.8499 & 0.708356 \\
\hline 0.35 & - & -1.213554 & - & -0.960658 & - & 0.390439 & - & 0.965354 \\
\hline 0.4 & - & -1.265207 & - & -1.030213 & - & 0.559130 & - & 2.027239 \\
\hline
\end{tabular}
viscoelastic parameter, i.e. $W e=0.3257864$ by implementing the algorithm followed by [9]. Furthermore, this table also shows that the horizontal component of skin friction $F^{\prime \prime}(0)$ increases on increasing the viscoelastic parameter Weissenberg number We for $a / c>1$ and an opposite result is obtained for $0<a / c<1$. It also indicates that for a fixed value of Weissenberg number We, horizontal skin friction component is an increasing function of stretching ratio parameter $a / c$. The influences of the $W e, a / c$ and slip parameter $\beta$ on the horizontal and shear components of skin friction are numerically presented in table 2 . It is observed that both $F^{\prime}(0)$ and $H^{\prime}(0)$ increase on increasing $W e$ and $a / c$, while they decrease with increase in slip $\beta$. 
Table 2. Effects of $W e, a / c$ and $\beta$ on $F^{\prime \prime}(0)$ and $H^{\prime}(0)$.

\begin{tabular}{ccccc}
\hline We & $a / c$ & $\beta$ & $F^{\prime \prime}(0)$ & $H^{\prime}(0)$ \\
\hline 0.1 & 1.2 & 0.5 & 0.240333 & 0.663479 \\
0.0 & & & 0.184739 & 0.615094 \\
0.1 & & & 0.240333 & 0.663479 \\
0.2 & & & 0.346492 & 0.745048 \\
0.3 & & & 0.626779 & 1.029077 \\
0.4 & & & 3.054070 & 1.953398 \\
& 0.1 & & -0.646816 & 0.194723 \\
& 0.5 & & -0.453255 & 0.558919 \\
& 1.1 & & 0.115922 & 0.656955 \\
& 1.2 & & 0.240333 & 0.663473 \\
& & 0.0 & 0.397435 & 1.064442 \\
& & 0.5 & 0.240333 & 0.663473 \\
& & 1 & 0.172978 & 0.483469 \\
& & 2 & 0.111109 & 0.313993 \\
& & 5 & 0.053710 & 0.153360 \\
\hline
\end{tabular}

Table 3. Effects of $W e, a / c, \beta, P r_{e f f}$ and $\lambda$ on $-\theta^{\prime}(0)$.

\begin{tabular}{|c|c|c|c|c|c|c|}
\hline$W e$ & $a / c$ & $\beta$ & $P r_{e f f}$ & $\lambda$ & $\alpha$ & $-\theta^{\prime}(0)$ \\
\hline 0.1 & 1.2 & 0.5 & 2 & 0.2 & 0.5 & 0.688739 \\
\hline 0.0 & & & & & & 0.688699 \\
\hline 0.1 & & & & & & 0.688739 \\
\hline 0.2 & & & & & & 0.713511 \\
\hline 0.3 & & & & & & 0.896275 \\
\hline \multirow[t]{22}{*}{0.4} & & & & & & 1.457372 \\
\hline & 0.1 & & & & & 0.401427 \\
\hline & 0.5 & & & & & 0.531463 \\
\hline & 1.1 & & & & & 0.670046 \\
\hline & 1.2 & & & & & 0.688739 \\
\hline & & 0.0 & & & & 0.677599 \\
\hline & & 1 & & & & 0.693268 \\
\hline & & 2 & & & & 0.697307 \\
\hline & & 5 & & & & 0.701461 \\
\hline & & & 0.5 & & & 0.444785 \\
\hline & & & 1 & & & 0.547677 \\
\hline & & & 2 & & & 0.688739 \\
\hline & & & 5 & & & 0.903157 \\
\hline & & & & -1 & & 0.951638 \\
\hline & & & & -0.5 & & 0.868039 \\
\hline & & & & 0.0 & & 0.751048 \\
\hline & & & & 0.5 & & 0.567194 \\
\hline & & & & 1 & & 0.205316 \\
\hline & & & & & 0 & 1.050499 \\
\hline & & & & & 1 & 0.512314 \\
\hline & & & & & 2 & 0.338762 \\
\hline & & & & & 5 & 0.168013 \\
\hline
\end{tabular}

Table 3 shows the influences of the Weissenberg number $W e$, stretching ratio parameter $a / c$, slip parameter $\beta$, effective Prandtl number $P r_{\text {eff }}$, heat source/sink parameter $\lambda$ and thermal slip parameter $\alpha$ on the effective local Nusselt number $-\theta^{\prime}(0)$. It is clearly seen that decrease in $N u_{x}^{*}$

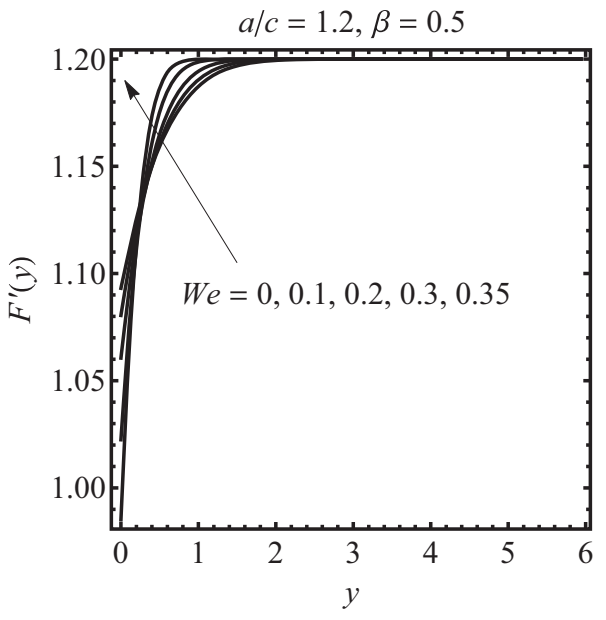

Figure 2. Effects of $W e$ on $F^{\prime}(y)$ when $a / c>1$.

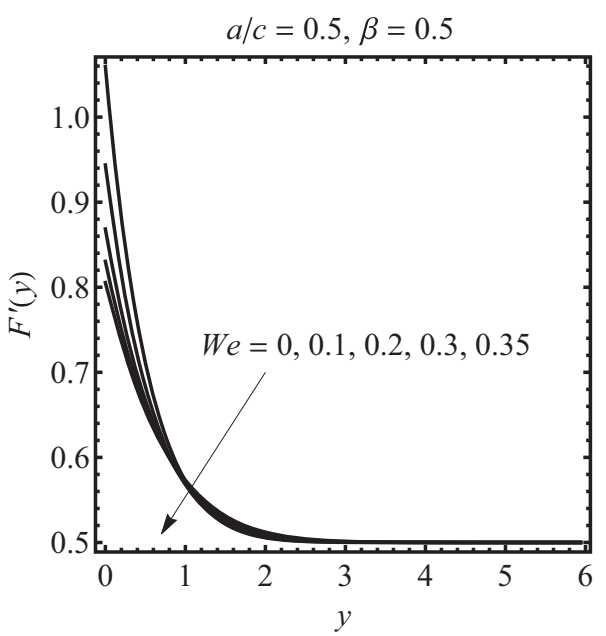

Figure 3. Effect of $W e$ on $F^{\prime}(y)$ when $a / c<1$.

occurs due to increase in $\lambda$ and $\alpha$. Other parameters (We, $a / c, \beta$ and $P r_{\text {eff }}$ ) show increasing effects.

Figures 2 and 3 show the influence of the viscoelastic parameter $W e$ on the dimensionless horizontal velocity $F^{\prime}(y)$ in the presence of slip effects for the stretching ratio parameter $a / c=1.2$, which correspond to the results that the stretching ratio parameter enhances the viscoelasticity effects. On the other hand, a increment in the Weissenberg number causes a decrease in the velocity profile for case $0<a / c<1$. It is worth mentioning that no phenomenon of overshoot (for $a / c>1$ ) and undershoot (for $a / c<1$ ) in the velocity profile is observed. Figure 4 shows the effects of stretching ratio parameter $a / c$ on the velocity profile. It is noted that the velocity increases for increasing $a / c$. Inverted boundary layer phenomenon is observed for $a / c<1$. Figures 5 and 6 indicate the effects of slip parameter on the velocity profile for both the cases. These figures show the 


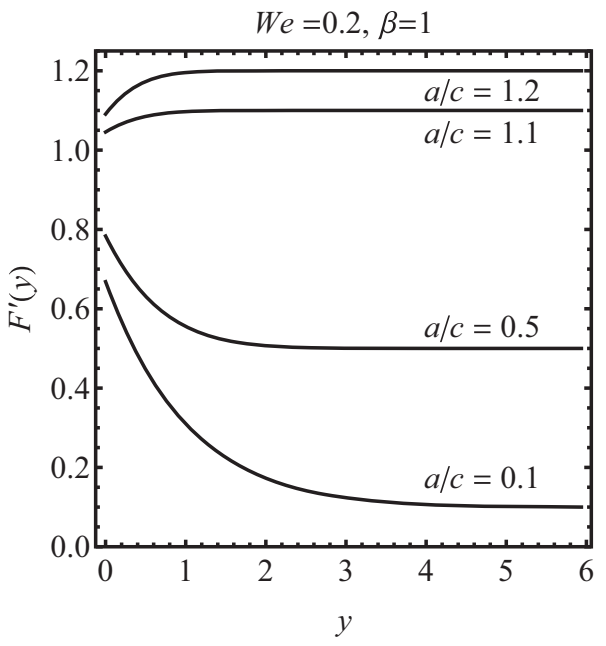

Figure 4. Effect of $a / c$ on $F^{\prime}(y)$.

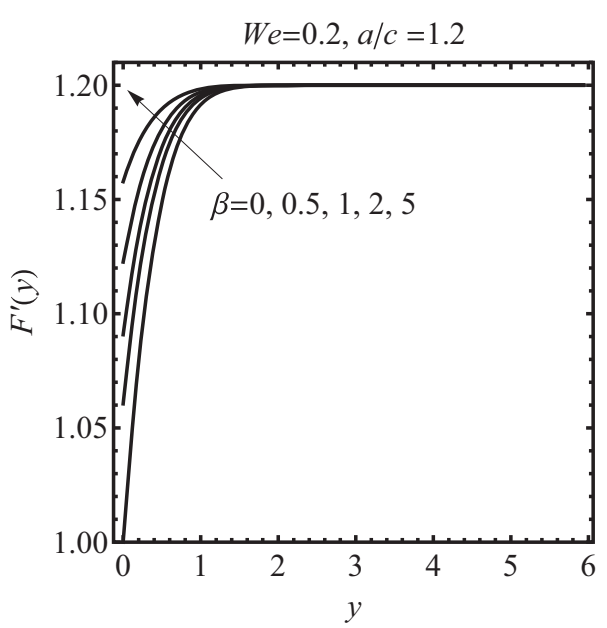

Figure 5. Effect of $\beta$ on $F^{\prime}(y)$ when $a / c>1$.

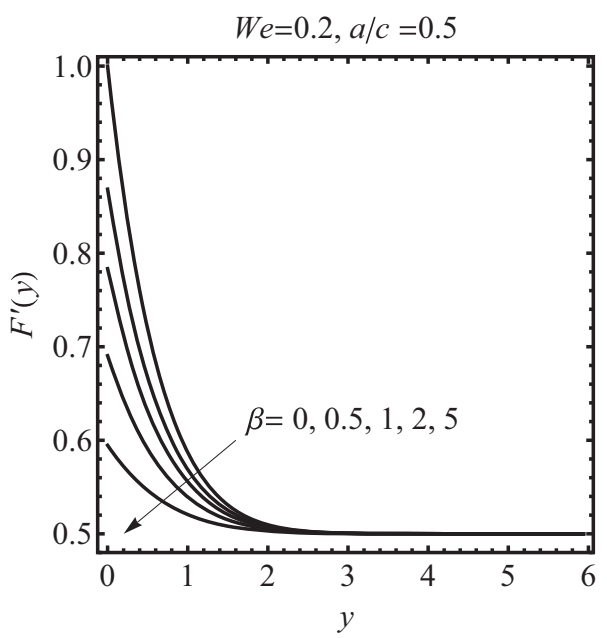

Figure 6. Effect of $\beta$ on $F^{\prime}(y)$ when $a / c<1$.

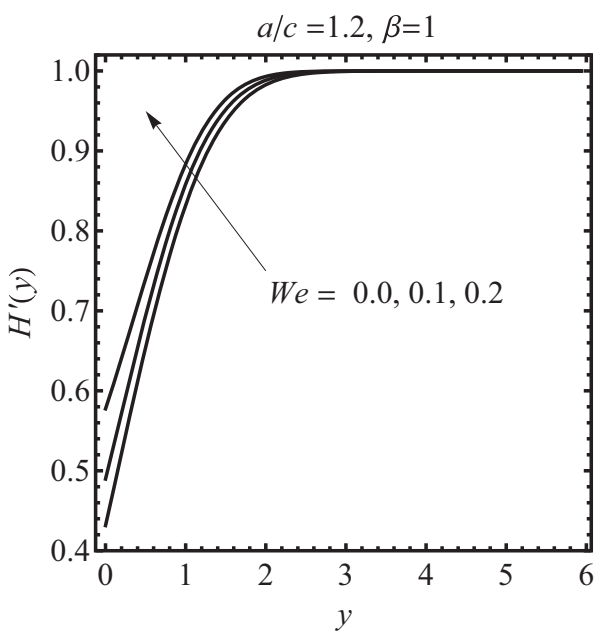

Figure 7. Effect of $W e$ on $H^{\prime}(y)$.

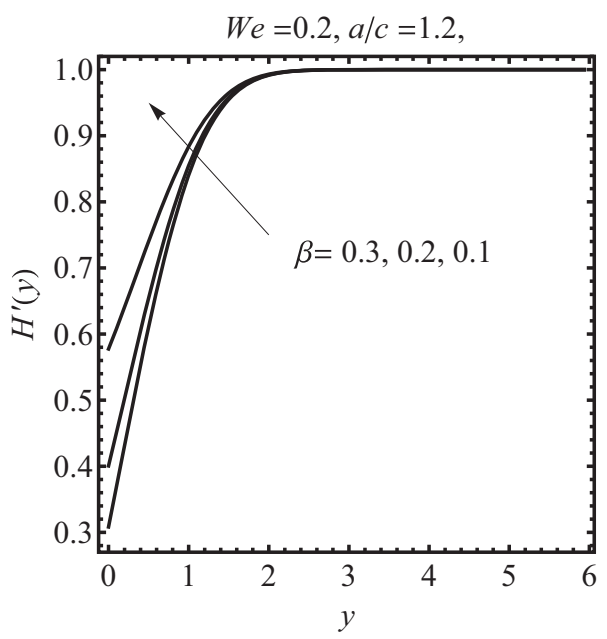

Figure 8. Effect of $\beta$ on $H^{\prime}(y)$.

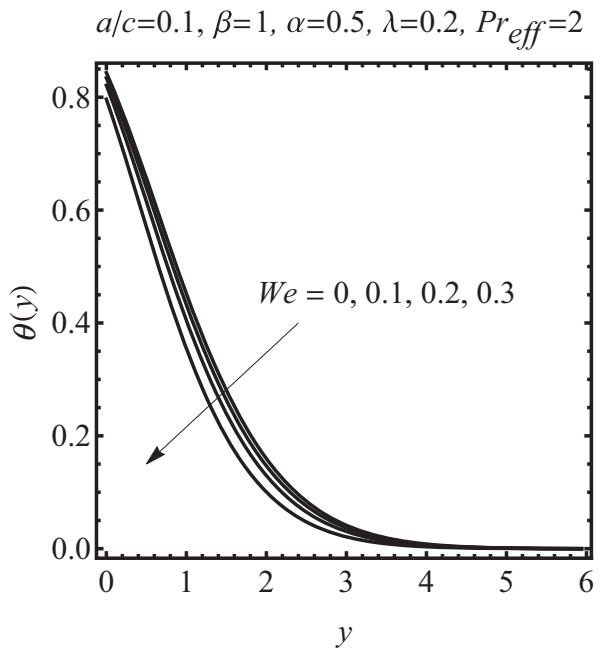

Figure 9. Effect of $W e$ on $\theta(y)$. 


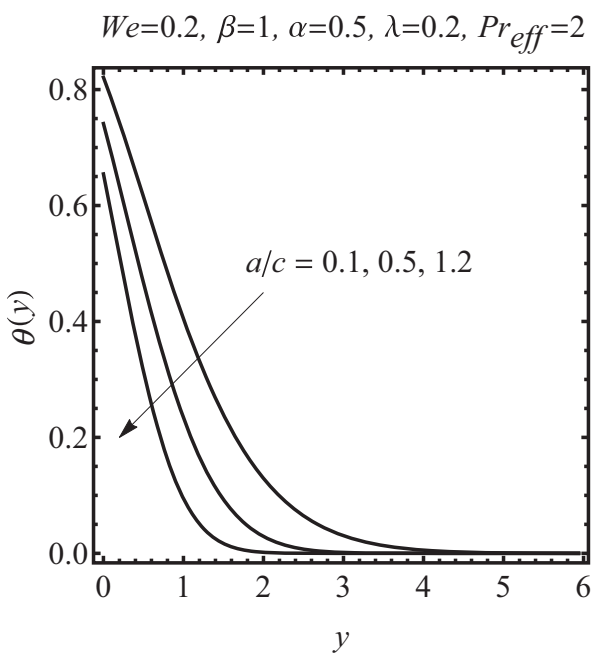

Figure 10. Effect of $a / c$ on $\theta(y)$.

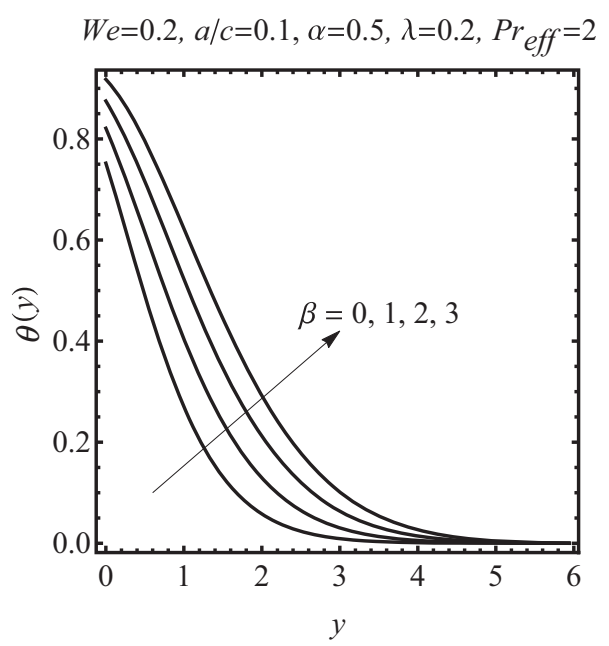

Figure 11. Effect of $\beta$ on $\theta(y)$.

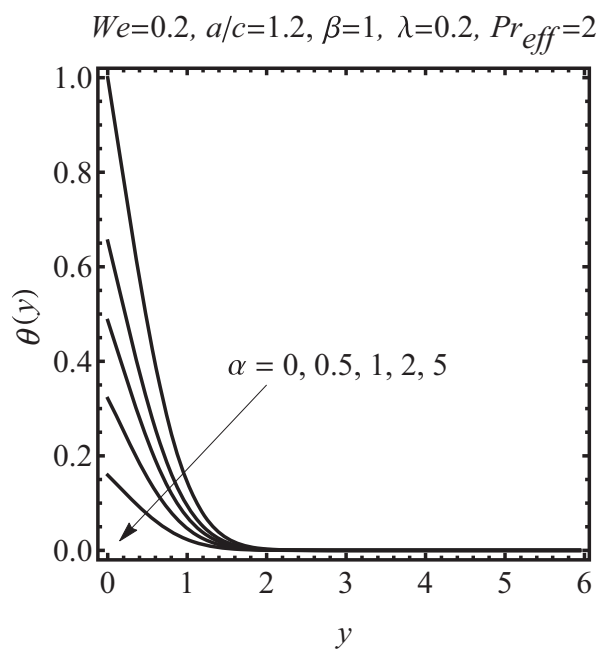

Figure 12. Effect of $\alpha$ on $\theta(y)$.

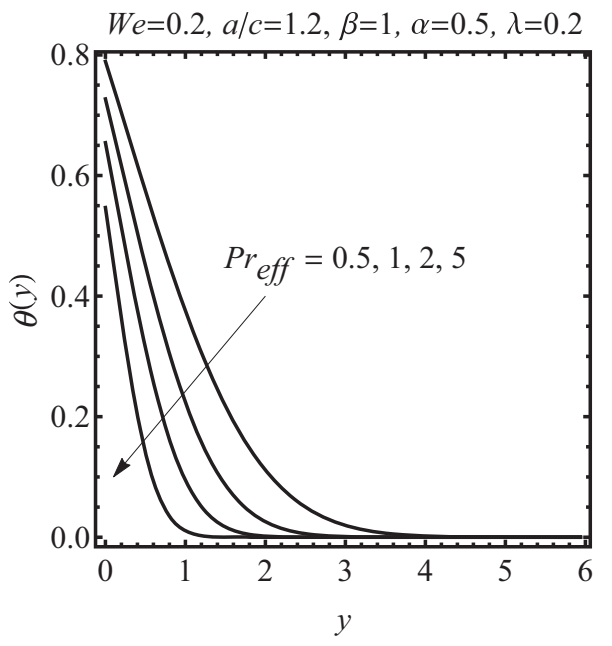

Figure 13. Effect of $P r_{e f f}$ on $\theta(y)$.

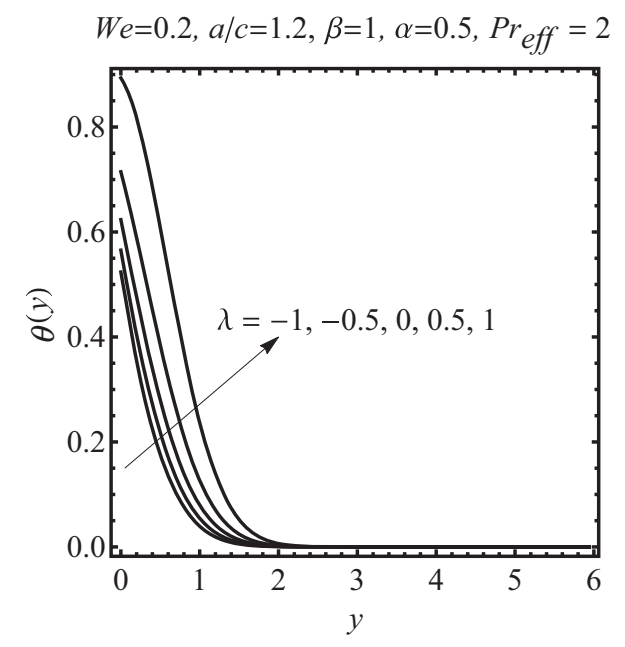

Figure 14. Effect of $\lambda$ on $\theta(y)$.

result that as slip is increased the velocity is increased for $a / c>1$ and decreased for $a / c<1$. The variation of shear component of skin friction $H^{\prime}(0)$ against $W e$ is plotted in figure 7. In the presence of slip, $H^{\prime}(0)$ is an increasing function of $W e$. Figure 8 presents the effects of slip parameter on $H^{\prime}(0)$. An increase in slip parameter results in a decrease of $H^{\prime}(0)$. Figure 9 analyses the viscoelastic effects on the dimensionless temperature profile $\theta$. Temperature and thermal boundary layer thickness decrease with increasing $W e$, resulting from the fact that the viscoelasticity enhances the heat transfer rate, which causes reduction in fluid temperature inside the boundary layer. Figure 10 presents the change in temperature profile for various values of $a / c$. It is noticed that increase in the stretching ratio parameter causes a decrease in the temperature profile, which results in the enhancement of the heat transfer rate. Figure 11 is plotted to check the effects 
of slip parameter on the temperature profile. Increase in the slip parameter enhances the temperature. The effects of thermal slip $\alpha$ on $\theta(y)$ is plotted in figure 12 . It can be seen that temperature inside the boundary layer decreases on increasing the thermal slip parameter. Figure 13 depicts the change in the temperature profile on changing the effective Prandtl number $P r_{\text {eff }}$. An increase in $P r_{\text {eff }}$ cause a decrease in temperature, which is due to the fact that thermal diffusivity reduces for large $P r_{\text {eff }}$. Figure 14 displays the heat source/sink effects on the temperature. Addition of heat source causes an increase in heat transfer rate, which results in an increase of temperature. On the other hand, addition of heat sink reduces the temperature.

\section{Conclusion}

- The undershoot and overshoot phenomenon in the velocity profiles within the boundary layer associated with the Walters-B fluid is controlled and a more accurate and stable solution is predicted.

- It is observed that an increase in viscoelasticity enhances the fluid velocity for $a / c>1$ and a reverse behaviour arises for $0<a / c<1$. The temperature and thickness of thermal boundary layer increase with rising viscoelasticity of the fluid.

- On increasing the stretching ratio parameter, the fluid velocity increases while temperature and thickness of thermal boundary layer decline. Inverted boundary layer phenomenon is observed for $a / c<1$.

- The presence of velocity slip enhances the velocity and temperature while an increment in thermal slip reduces the temperature.

- Large value of effective Prandtl number reduces the temperature within the boundary layer.

- Addition of heat source increases the temperature while addition of heat sink reduces the temperature.

\section{Nomenclature}

$\bar{u} \quad$ velocity component of fluid

$\bar{v} \quad$ velocity component of fluid

$\bar{x} \quad$ rectangular coordinate

$\bar{y} \quad$ rectangular coordinate

$\bar{p} \quad$ fluid pressure

$k_{0} \quad$ material parameter

$c_{p} \quad$ specific heat at constant pressure

$q_{r} \quad$ radiative heat flux

$Q \quad$ heat source sink

$T \quad$ temperature of fluid

$T_{w}, T_{\infty} \quad$ surface and Ambient tempreatures

$A \quad$ boundary layer displacemnt

We Weissenberg number

$\begin{array}{ll}C_{f} & \text { skin friction coeffient } \\ q_{w} & \text { heat flux at wall } \\ \operatorname{Re}_{x} & \text { local Reynold number } \\ \operatorname{Pr}_{e f f} & \text { effective Prandtl number } \\ N u_{x}^{*} & \text { effective Nusselt number } \\ \mu & \text { dynamic viscosity } \\ v & \text { kinametic viscosity } \\ \rho & \text { density } \\ \tau_{w} & \text { wall shear stress } \\ \alpha & \text { thermal slip parameter } \\ \beta & \text { velocity slip parameter } \\ \lambda & \text { heat source sink parameter } \\ \gamma & \text { shear in freestream } \\ \psi & \text { stream function }\end{array}$

\section{References}

[1] Beard D W and Walters K 1964 Elastico-viscous boundary layer flows. I. Two-dimensional flow near a stagnation point. Proc. Camb. Philos. Soc. 60: 667-674

[2] Frater K R 1970 On the solution of some boundary value problems arising in elasticoviscous fluid mechanics. $Z$. Angew. Math. Phys. 2: 134-137

[3] Ariel P D 1992 A hybrid method for computing the flow of viscoelastic fluids. Int. J. Numer. Meth. Fluids 14: 757-774

[4] Dorrepaal J M, Chandna O P and Labropulu F 1992 The flow of a viscoelastic fluid near a point of reattachment. Z. Angew. Math. Phys. 43: 708-714

[5] Crane L J 1970 Flow past a stretching plate. Z. Angew. Math. Phys. 21: 645-647

[6] McLeod J B and Rajagopal K R 1987 On the uniqueness of flow of a Navier-Stokes fluid due to a stretching boundary. Arch. Ration. Mech. Anal. 98: 385-393

[7] Mahapatra T R, Dholey S and Gupta A S 2007 Oblique stagnation point flow of a viscoelastic fluid towards a stretching surface. Int. J. Nonlin. Mech. 42: 484-499

[8] Lok Y Y, Amin N and Pop I 2006 Non-orthogonal stagnation point flow towards a stretching sheet. Int. J. Nonlin. Mech. 41: $622-627$

[9] Hussain I, Labropulu F and Pop I 2011 Two dimensional oblique stagnation point flow towards a stretching surface in a viscoelastic fluid. Cent. Eur. J. Phys. 9: 176-182

[10] Hayat T, Sajid M and Pop I 2008 Three-dimensional flow over a stretching surface in a viscoelastic fluid. Nonlin. Anal. Real World Appl. 9: 1811-1822

[11] Wang C Y 2003 Stagnation flows with slip: exact solution of the Navier-Stokes equations. Z. Angew. Math. Phys. 54: 184-189

[12] Labropulu F, Hussain I and Chinichian M 2004 Stagnation point flow of Walters B fluid with slip. Int. J. Math. Math. Sci. 61: 3249-3258

[13] Sajid M, Mehmood K and Abbas Z 2012 Axisymmetric stagnation-point flow with a general slip boundary condition over a lubricated surface. Chin. Phys. Lett. 29: 024702

[14] Mehmood K, Sajid M and Ali N 2016 Non-orthogonal stagnation point flow of a second grade fluid past a lubricated surface. Z. Naturforsch. A 71: 273-280 
[15] Mahmood K, Sajid M, Ali N and Sadiq M N 2017 Effects of lubricated surface in the oblique stagnation point flow of a micropolar fluid. Eur. Phys. J. Plus. 132: 297

[16] Mahmood K, Sajid M, Sadiq M N and Ali N 2018 Effects of lubrication on the steady oblique stagnation-point flow of a couple stress fluids. Phys. Astron. Int. J. 2: 389-397

[17] Mahmood K, Sadiq M N, Sajid M and Ali N 2019 Heat transfer in stagnation-point flow of a Jeffrey fluid past a lubricated surface. J. Braz. Soc. Mech. Sci. Eng. 41: 65

[18] Kushwaha H M and Sahu S K 2016 Analysis of slip flow heat transfer between two unsymmetrically heated parallel plates with viscous dissipation. Sadhana 41: 653-666

[19] Zhu J, Zheng L, Zheng L and Zhang X 2015 Second-order slip MHD flow and heat transfer of nanofluids with thermal radiation and chemical reaction. Appl. Math. Mech. Engl. Ed. 36: 1131-1146

[20] Dandapat B S and Gupta A S 1989 Flow and heat transfer in a viscoelastic fluid over a stretching sheet. Int. J. Nonlin. Mech. 24: 215-219

[21] Sajid M, Sadiq M N, Ali N and Javed T 2018 Numerical simulation for Homann flow of a micropolar fluid on a spiraling disk. Eur. J. Mech. B Fluids 72: 320-327

[22] Labropulu F, Li D and Pop I 2010 Non-orthogonal stagnation point flow towards a stretching surface in a non-Newtonian fluid with heat transfer. Int. J. Therm. Sci. 49: $1042-1050$

[23] Reza M, Panigrahi S and Mishra A K 2017 Stagnation point flow and heat transfer for a viscoelastic fluid impinging on a quiescent fluid. Sadhana 42: 1979-1986

[24] Hayat T, Asad S, Mustafa M and Alsulami H H 2014 Heat transfer analysis in the flow of Walters' B fluid with convective boundary condition. Chin. Phys. $B$ 23: 084701

[25] Abdul Hakeem A K, Ganesh N V and Ganga B 2014 Effects of heat radiation in Walter's liquid B fluid over a stretching sheet with non-uniform heat source/sink and elastic deformation. J. King Saud Univ. Eng. Sci. 26: 168-175
[26] Ali N, Khan S U and Abbas Z 2015 Unsteady flow of third grade fluid over an oscillatory stretching sheet with thermal radiation and heat source/sink. Nonlin. Eng. 1-14

[27] Seddeek M A and Abdelmeguid M S 2006 Effects of radiation and thermal diffusivity on heat transfer over a stretching surface with variable heat flux. Phys. Lett. A 348: 172-179

[28] Babu M J and Sandeep N 2016 Effect of nonlinear thermal radiation on non-aligned bio-convective stagnation point flow of a magnetic-nanofluid over a stretching sheet. Alex. Eng. J. 55: 1931-1939

[29] Raju C S K and Sandeep N 2017 MHD slip flow of a dissipative Casson fluid over a moving geometry with heat source/sink: a numerical study. Acta Astronaut. 133: 436-443

[30] Kumar K A, Sugunamma V and Sandeep N 2018 Impact of non-linear radiation on MHD non-aligned stagnation point flow of micropolar fluid over a convective surface. J. NonEqu. Thermodyn. 43: 327-345

[31] Attia H A, Abbas W, Abdeen M A M and Said A A M 2015 Heat transfer between two parallel porous plates for Couette flow under pressure gradient and Hall current. Sadhana 40: 183-197

[32] Prakashi O, Makinde O D, Kumar D and Dwived Y K 2015 Heat transfer to MHD oscillatory dusty fluid flow in a channel filled with a porous medium. Sadhana 40: 1273-1282

[33] Magyari E and Pantokratoras A 2011 Note on the effect of thermal radiation in the linearized Rosseland approximation on the heat transfer characteristics of various boundary layer flows. Int. Commun. Heat Mass Transfer 38: 554-556

[34] Yuan J Y, Hua W, Ping M H and Yu G B 2011 Multidomain pseudospectral methods for nonlinear convectiondiffusion equations. Appl. Math. Mech. Engl. Ed. 32: $1255-1268$

[35] Sajid M, Iqbal S A and Ali N 2017 A Legendre wavelet spectral collocation technique resolving anomalies associated with velocity in some boundary layer flows of Walter-B liquid. Meccanica 52: 877-887 